University of Nebraska - Lincoln

DigitalCommons@University of Nebraska - Lincoln

January 2007

\title{
Nanoscale Characterization of Electronic and Electrical Properties of III-Nitrides by Scanning Probe Microscopy
}

Brian J. Rodriguez

Oak Ridge National Laboratory, brian.rodriguez@ucd.ie

Alexei Gruverman

University of Nebraska-Lincoln, agruverman2@unl.edu

Robert J. Nemanich

North Carolina State University, robert.nemanich@asu.edu

Follow this and additional works at: https://digitalcommons.unl.edu/physicsgruverman

Part of the Physics Commons

Rodriguez, Brian J.; Gruverman, Alexei; and Nemanich, Robert J., "Nanoscale Characterization of Electronic and Electrical Properties of III-Nitrides by Scanning Probe Microscopy" (2007). Alexei Gruverman Publications. 45.

https://digitalcommons.unl.edu/physicsgruverman/45

This Article is brought to you for free and open access by the Research Papers in Physics and Astronomy at DigitalCommons@University of Nebraska - Lincoln. It has been accepted for inclusion in Alexei Gruverman Publications by an authorized administrator of DigitalCommons@University of Nebraska - Lincoln. 
Published in:

Scanning Probe Microscopy: Electrical and Electromechanical Phenomena at the Nanoscale, Sergei Kalinin and Alexei Gruverman, editors, 2 volumes (New York: Springer Science+Business Media, 2007).

$\begin{array}{lllllll}\text { D } & \mathbf{0} & \mathbf{0} & \mathbf{0} & \mathbf{0} & \mathbf{0} & \mathbf{0}\end{array}$

Brian J. Rodriguez, Oak Ridge National Laboratory Alexei Gruverman, University of Nebraska-Lincoln

Robert J. Nemanich, North Carolina State University 


\title{
Nanoscale Characterization of Electronic and Electrical Properties of III-Nitrides by Scanning Probe Microscopy
}

\author{
B. J. Rodriguez, A. GRUverman, AND R. J. NEMANich
}

Recent interest in the technological potential of nitride-based semiconductors has led to the development and commercialization of a wide range of electronic devices. The nanoscale investigation of the electric properties of III-nitride thin films, bulk crystals, and heterostructures is of considerable interest for determining how interfaces, defects, and inversion domain boundaries affect device performance. The pyroelectric nature of wurtzitic III-nitrides is characterized by a spontaneous polarization that exists without the presence of an external field and by a polarization-bound surface charge. Scanning probe-based measurements of surface contact potentials and surface band bending in these materials, which are of crucial importance to device design, are summarized in this review chapter. In addition, the role of charged defects on device performance is explored by scanning probe techniques. Lastly, the measurement of polarity and of the screening mechanism of III-nitrides, which are fundamental issues for the fabrication of devices based on polarity effects, are discussed. This chapter provides a critical analysis of the contributions made by scanning probe-based techniques toward a deeper understanding of the III-nitride material system.

\section{Introduction}

The large energy band gap, high thermal conductivity, and stability at high temperatures exhibited by GaN have generated significant interest in employing the III-nitride system ( $\mathrm{GaN}, \mathrm{AlN}$, and $\mathrm{InN}$ ) for the fabrication of modern micro and nano-electronic and optoelectronic devices. In addition, the use of ternary and quaternary alloys facilitates band gap and lattice constant tunability, while the pyroelectric and piezoelectric properties of wurtzitic III-nitrides provide an added dimension to device design, enabling the III-nitride system to establish itself as a formidable technological platform [1]. In fact, many GaN-based semiconductor devices such as blue and white light-emitting diodes, blue-emitting lasers, high-power and/or high-frequency transistors, surface acoustic wave devices, and sensors are currently under development or in production [2]. The useful materials properties of GaN have their own challenges; and, as with any material system, 
TABLE 1. Polarization, Polarization Charge Density, Band Gap, and Piezoelectric Coefficients for AlN, GaN, and InN

\begin{tabular}{lcccccc}
\hline Material & $\begin{array}{c}P_{S P}[3] \\
\left(\mathrm{C} / \mathrm{m}^{2}\right)\end{array}$ & $\begin{array}{c}\sigma / e \\
\left(\mathrm{~cm}^{-2}\right)\end{array}$ & $E_{g}(\mathrm{eV})$ & $\begin{array}{c}d_{33}[6] \\
(\mathrm{pm} / \mathrm{V})\end{array}$ & $\begin{array}{c}d_{31}[6] \\
(\mathrm{pm} / \mathrm{V})\end{array}$ & $\begin{array}{c}d_{15}[6] \\
(\mathrm{pm} / \mathrm{V})\end{array}$ \\
\hline AIN & -0.090 & $5.6 \times 10^{13}$ & $6.2[4]$ & 5.4 & -2.1 & 2.9 \\
$\mathrm{GaN}$ & -0.034 & $2.1 \times 10^{13}$ & $3.4[4]$ & 2.7 & -1.4 & 1.8 \\
InN & -0.042 & $2.6 \times 10^{13}$ & $0.7[5]$ & 7.6 & -3.5 & 5.5 \\
\hline
\end{tabular}

technical obstacles must be overcome. Some of these obstacles and challenges have been addressed by scanning probe-based techniques, which is the focus of this chapter. In particular, the role of polarization in III-nitride device design is explored.

The measurement of the dielectric properties of III-nitrides with high spatial resolution is of importance for the design and performance of novel electronic and optoelectronic devices, particularly those based on polarization effects. At the surfaces of GaN crystals (spontaneous polarization, $P_{S P}=-0.034 \mathrm{C} / \mathrm{m}^{2}$ ), for example, a divergence in the spontaneous polarization induces a polarizationbound surface charge with a density of $2.1 \times 10^{13} \mathrm{~cm}^{-2}$ [3]. The sign of the polarization-induced charge at each surface is related to the orientation of the polarization. Values for polarization and bound charge density for $\mathrm{GaN}, \mathrm{AlN}$, and InN are presented in Table 1.

While spontaneous polarization is independent of strain, piezoelectric polarization $P_{P E}$ is strain-induced, and the total polarization of a nitride layer is the sum of the spontaneous and piezoelectric polarizations, $P_{\text {total }}=P_{S P}+P_{P E}$ [7]. Alternatively, the piezoelectric polarization can be considered as a modification of the spontaneous polarization. Based on symmetry, a nonvanishing polarization is allowed in an ideal wurtzite $(c / a=1.63299, u=0.375$, where $u$ is the anioncation bond length along the [0001] axis [8]). For example, as $c / a$ decreases and $u$ increases (as the nonideality of crystal structure increases as a result of strain), $P_{S P}$ increases. The orientation of the spontaneous polarization is defined by convention to be parallel to the crystallographic [0001] axis [9]. Ga-face $\mathrm{GaN}$ is oriented along the [0001] direction (and has spontaneous polarization along the [000]] direction) while $\mathrm{N}$-face GaN is oriented along the [0001] direction [10]. The wurtzite crystal structure of GaN is shown in Figure 1(a) and a schematic of the polarization and of the screening charge is shown in Figure 1(b). In Figure 2(a) a schematic demonstrating the internal electric field due to spontaneous polarization is presented, and in Figure 2(b), the band structure when free carriers are taken into account is shown. In the latter case, free carriers and ionized donors screen the polarizationbound charge. For epitaxial layers of $\mathrm{GaN}$ with $\mathrm{Ga}$-face polarity, the bound surface charge is negative, whereas for $\mathrm{N}$-face $\mathrm{GaN}$, the bound surface charge is positive. At the free surface it is expected that internal charge (free carriers, ionized donors or acceptors, and charged defects) and external charge (adsorbed molecules or surface reconstruction) will act to screen the bound polarization charge. This charge is shown schematically in Figure 1(b). Charged surface states can also contribute to 
(a)

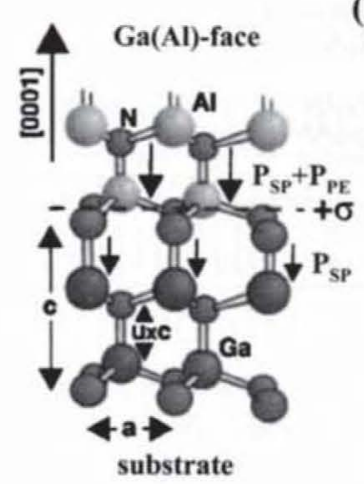

(b)

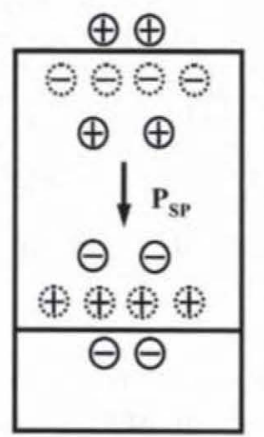

Adsorbed charge

Polarization bound charge

Free carrier charge

Spontaneous polarization

Free carrier charge

Polarization bound charge

Substrate screening charge

FIGURE 1. (a) Wurtzite crystal structure and (b) schematic of polarization and screening charge for $\mathrm{Ga}(\mathrm{Al})$-face $\mathrm{Ga}(\mathrm{Al}) \mathrm{N}$. Figure 1(a) reprinted with permission from [6]. Copyright 1999, American Institute of Physics.

screening and additionally affect band bending. For example, the negative bound charge at the $n$-type GaN Ga-face surface is screened by positively ionized donors, leading to upward band bending and a depletion region at the surface [11]. The positive bound surface charge at the $\mathrm{N}$-face surface would give rise to a free electron accumulation layer, which would fix the Fermi level near the conduction band edge. This results in a slight downward bending of the conduction band edge at a clean $\mathrm{N}$-face surface.

The polarization and screening of the III-nitrides adds complexity that can either be used advantageously, as with polarization-dependent devices such as the twodimensional electron gas (2DEG) high electron mobility transistor (HEMT), or that can yield unwanted complications. Devices such as HEMTs or quantum wells are both based on heterostructures. Lattice mismatch between layers can introduce piezoelectric strain and cause the interface charge to be distributed nonuniformly.

(a)

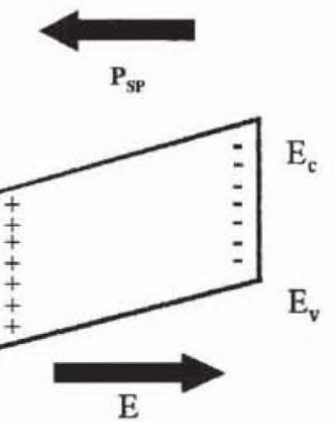

(b)

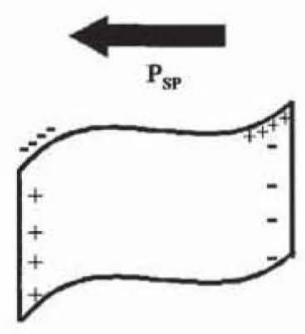

FIGURE 2. (a) Schematic demonstrating the internal electric field due to spontaneous polarization and (b) the band structure when free carriers are taken into account. The free carriers screen the polarization bound charge. 
Functional devices require understanding and controlling charge at surfaces and interfaces, and in order to gain further insight into the condition of the surface, nanoscale electrical measurements are required.

Scanning probe microscopy (SPM) has proven itself to be a valuable tool for III-nitride characterization. In this review, we limit the discussion to electrical characterization on the nanoscale. When a conducting tip is near a semiconducting surface, the tip interacts with the charge on the surface. Depending on the voltage applied to the tip, it is possible to modify and measure the charge and or surface potential distribution on the surface. When the tip is in contact with the surface and a field is applied through the bulk of the sample, it is possible to measure the electromechanical response of the sample. SPM-based techniques have been employed to perform high-resolution characterization of the local electronic properties of the III-nitrides [12-51]. Local electronic properties of III-nitrides were first studied by Hansen et al. [12] in 1998. They employed scanning capacitance microscopy (SCM) to investigate threading dislocations in $\mathrm{GaN}$. Since then, SCM [13-15], electrostatic force microscopy (EFM) [16-20], Kelvin probe force microscopy (KPFM) [18-36],conductive-tip atomic force microscopy (C-AFM) [35-46], piezoresponse force microscopy (PFM) [47-50] and scanning gate microscopy (SGM) [51] have all been employed to characterize III-nitride films and surfaces.

One of the main advantages of using SPM for electrical characterization of IIInitrides is that the measurements are performed on the micro- to nanoscale, which is the relevant dimension of actual devices. In addition, these techniques allow nanoscale measurements of the same properties (i.e., polarization, piezoelectricity) that make the devices functional. Not only can these fundamental issues be addressed, but so also can issues that are important to device performance.

\section{Characterization of Surface Electronic Properties by SPM}

In this section we explore the use of EFM and KPFM to measure electronic properties of III-nitrides. In particular, we describe the use of EFM and KPFM for the determination of surface potentials, band bending, and surface state densities and how these parameters relate to device design. While detailed descriptions of EFM [52,53] and KPFM [54] can be found elsewhere, including this book, here we provide a brief background to facilitate useful discussion.

There are several approaches to implementing EFM and KPFM; some techniques utilize a mechanically driven cantilever (force gradient technique) while others utilize an electrically driven cantilever (voltage modulation). The force gradient technique employs a lift height approach, in which the cantilever first measures topography, and then retraces the same line at a certain lift height to measure only the electrostatic interaction, which manifests itself as a shift in the resonant frequency of the tip. The voltage modulation approach developed by Terris et al. [52] and Saurenbach et al. [53] uses both modulations (mechanically 
driven cantilever and voltage modulation applied to the tip), allowing topographic and electrostatic images to be acquired simultaneously.

In EFM and KPFM, the conducting tip is held anywhere from 10-100 nm above the sample surface (where electrostatic interactions dominate) while both a dc and an ac voltage are applied to the tip. In EFM, the force between the tip and surface is a combination of electrostatic and capacitive forces. The EFM image (this mode of EFM is also called open-loop KPFM) is constructed from the first harmonic $(1 \omega)$ component of this force [55],

$$
F_{1 \omega}=Q_{t} E_{s}+\frac{\partial C_{t}}{\partial z}\left(V_{d c}-V_{s}\right) V_{a c},
$$

where $Q_{t}=C_{t} V_{a c}$ (first harmonic) is the charge on the tip, $C_{t}$ is the capacitance of the tip-surface configuration, $E_{s}=\sigma / \varepsilon_{0}(1+\kappa)$ is the field due to an infinite sheet of uniform charge $\sigma, \kappa=9.5$ is the dielectric constant of $\mathrm{GaN}, \partial C_{t} / \partial z$ is the partial derivative of the tip-surface capacitance with respect to the tip-surface separation, $V_{d c}$ and $V_{a c}$ are the dc and ac voltages applied to the tip, and $V_{s}$ is the surface potential. The surface potential can be expressed as [16,56]

$$
V_{s}=\frac{1}{e}\left(\phi_{m}-\chi_{s}-\Delta E_{f n}-\Delta \phi\right),
$$

where $\phi_{m}$ is the metal work function of the tip coating, $\chi_{s}$ is the electron affinity of the surface, $\Delta E_{f n}$ is the position of the Fermi level in the bulk with respect to the conduction band, and $\Delta \phi$ is the band bending. The surface potential, $V_{s}$, or contact potential difference, is shown schematically in Figure 3.

KPFM employs a dc feedback loop, which varies the dc bias such that the amplitude of the $1 \omega$ tip vibration is minimized. This value of dc bias that minimizes the $F_{1 \omega}$ signal (Eq. (1).) is equal to the surface potential $\left(V_{s}\right)$, and by recording this value, an image of the surface potential can be constructed.

FIGURE 3. Electronic band structure of GaN showing schematically the surface potential in relation to the workfunction difference between metal and semiconductor.

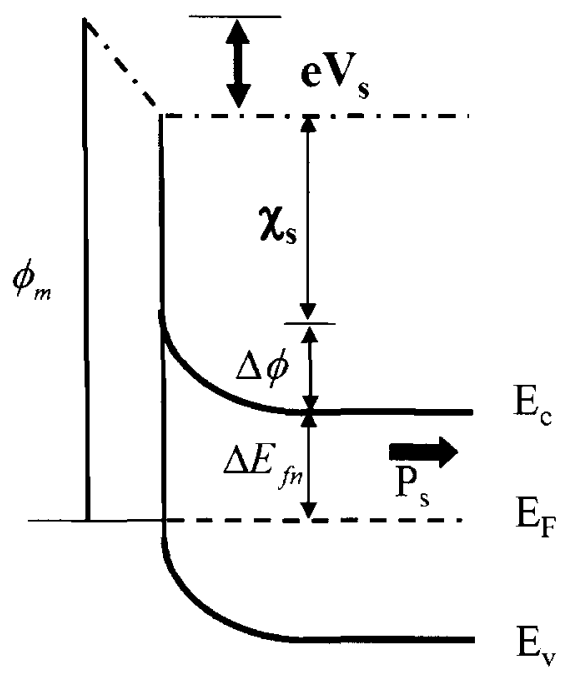




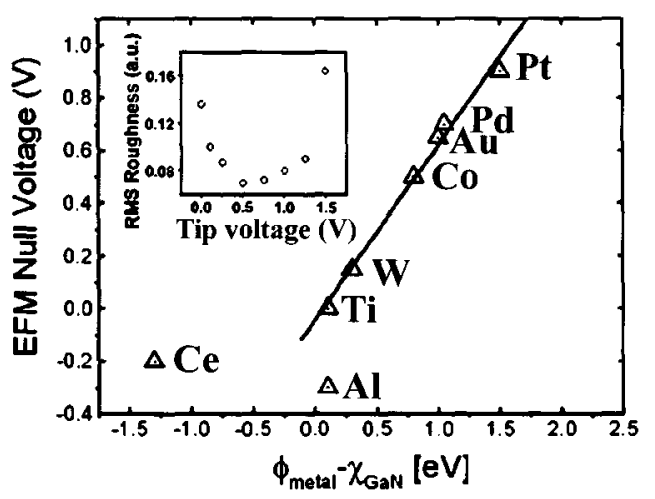

FIGURE 4. A plot of the tip voltage that minimized the electrostatic force versus the work function difference between the metal coated tip and the GaN surface. Reprinted with permission from [16]. Copyright 1999, American Institute of Physics.

With EFM, the measured electrostatic force on the tip can be directly related to the net charge at the surface. This net surface charge is a combination of bound charge, internal screening charge, and external screening (adsorbed) charge. While EFM is often considered a qualitative measurement, the technique can be used to determine the sign of the charge, and the magnitude of the charge can be determined by modeling the tip-sample capacitance. The complexity of the models varies from a simple point charge to a more rigorous model which takes into account the conical tip shape and electrostatic forces on the cantilever beam [57].

EFM has been used extensively for the observation of polarity contrast between $\mathrm{Ga}$ - and $\mathrm{N}$-face $\mathrm{GaN}$ (EFM for polarity imaging is discussed later in this chapter) Few attempts have been made to quantify surface charge for the reasons previously stated. However, in 1999, in one of the first SPM papers on electrical characterization of III-nitrides, Bridger et al. [16] used EFM to measure the local variation of piezoelectrically induced surface charge, contact potentials, and surface states in GaN. The measurements used tips of different metal coatings (therefore, with different metal work functions) and measured the dc bias that nullified the EFM signal. In this manner, they were able to determine the density of surface states. A plot of the tip voltage that minimized the electrostatic force versus the work function difference between the metal coated tip and the GaN surface is shown in Figure 4 . They model the situation as a metal tip separated from a sample by a dielectric interface region (they assume a water layer) equal to the distance between the tip and a semiconductor surface with surface states up to a particular energy. From this electrostatic analysis of the slope and intercept of the least squares linear fit, the density of surface states is $(7.5 \pm 0.5) \times 10^{12} \mathrm{~cm}^{-2}$, located $30 \mathrm{mV}$ above the valence band [16].

EFM has also been employed to explore the effect of doping on GaN films. Shmidt et al. [17] employed EFM to observe a reduction in EFM contrast variation in Si-doped GaN epilayers when compared to undoped samples [17]. They suggest Si doping suppresses the formation of point defects in GaN films.

KPFM is considered a quantitative measurement as opposed to EFM since it is a measurement of the contact potential difference between tip and sample, and therefore can be used to study the band structure of the material under investigation. It is 
a particularly valuable tool when combined with other techniques (i.e. techniques that can measure band gap or band bending). KPFM alone can be used to obtain $V_{s}$, however, when other parameters are known, Eq. (2) can be used to determine, for example, the electron affinity (when the band gap and the band bending are known) or band bending (when the electron affinity and the band gap are known).

KPFM has been used to measure surface (contact) potentials [18-36] as a function of polarity, doping and surface treatment, surface band bending [22], and bare surface barrier heights [24] of III-nitrides. The measurement of these electronic properties is critical for designing devices based on III-nitrides. As discussed in the introduction, III-nitrides in the wurtzite phase have a spontaneous polarization that results in a surface bound charge. The surface bound charge results in an electric field that can substantially affect the formation of a stable heterojunction interface and can cause surface band bending. The polarization-induced surface charge is balanced by a bound charge of opposite sign at the nitride/substrate interface [58]. These polarization charge densities (which are opposite in sign for $\mathrm{Ga}$ - and $\mathrm{N}$-face $\mathrm{GaN}$ surfaces) have been found to affect the local surface properties. Futhermore, the polarization and screening charges can influence the band bending. The direction and amount of band bending depends intrinsically on the polarity of the material and is reflected in KPFM studies of GaN surfaces. For example, Jones et al. [19] measured a surface potential difference between $\mathrm{Ga}$ - and N-face GaN of $\sim 55 \pm 10 \mathrm{mV}$. Cimalla et al. [25] reported a potential drop of $\sim 0.1 \mathrm{~V}$ across an inversion domain boundary from the $\mathrm{N}$ - to Ga-face side in a GaN lateral polarity heterostructure (LPH) sample. Recently, Rodriguez et al. [20] measured a surface potential difference of $0.6 \mathrm{~V}$ between $\mathrm{Ga}$ - and N-face regions of a GaN-LPH sample. The discrepancies in the magnitude of the potential differences between polar faces could be due to variations in the sample or surface conditions. The magnitude of the surface potential, however, also depends on the dopant type and concentration. This information is important for device design since not only do the measurements indicate that the surface potential is typically higher for $n$-type $\mathrm{GaN}$, but they demonstrate that opposite polar faces have different band-bending and, therefore, different electronic properties.

Using KPFM to measure the contact potential difference and assuming the electron affinity of $\mathrm{GaN}$ to be $3.5 \pm 1.0 \mathrm{eV}$, Koley et al. [24] calculated the bare surface barrier height (BSBH) of GaN films and $\mathrm{AlGaN} / \mathrm{GaN}$ heterostructures in an ambient environment. They reported that the BSBH decreased from $\sim 1.4$ to $\sim 0.6 \mathrm{eV}$ with increased $n$-type doping in Ga-face $\mathrm{GaN}$ [13]. From these measurements, the surface state density was determined to be $\sim 2.0 \times 10^{13} \mathrm{~cm}^{-2} \mathrm{eV}^{-1}$ located $0.70 \mathrm{eV}$ below the conduction band. Differences in surface state density between Refs. 16 and 24 can be attributed to differences in sample growth and preparation, and stress the importance of vacuum measurements. In addition, Koley et al. [24] found that the semiconductor work function of AlGaN decreased by $\sim 0.60$ $\mathrm{eV}$ with an increase in AlGaN thickness from $\sim 50$ to $440 \AA$ in AlGaN/GaN heterostructure samples. This decreasing trend in work function was explained by the presence of a uniform layer of negatively charged acceptors which act to screen the polarization. 
A dopant-dependant difference of the surface potential has been reported by da Silva et al. [26]. In this study, a cross-sectioned sample of GaN/AlGaN p-n heterostructures was utilized. EFM and KPFM measurements yielded a potential drop of $0.22 \mathrm{~V}$ between the $\mathrm{n}$ - and p-type regions of the $\mathrm{GaN}$ cross-section. Using the value reported by Koley et al. [24] of $0.6 \mathrm{eV}$ for heavily doped n-type $\mathrm{GaN}$, da Silva et al. [26] calculated that the band bending of the p-type material must be higher than $1.6 \mathrm{eV}$. This is consistent with $\mathrm{x}$-ray photoelectron spectroscopy and ultraviolet photoelectron spectroscopy measurements [59].

The measurement of surface potential may reflect the preparation and growth conditions used, and thus only apply to the particular sample being measured. More general information can be obtained by subjecting the surface to a well-controlled surface treatment, for example, a common cleaning technique. In this case, the difference between the surface potentials before and after the cleaning yields detailed information about the state of the surface and the electronic properties of the material.

In 2002, Hsu et al. [21] also measured surface contact potential variations of GaN surfaces by KPFM. They reported that the surface contact potential varied from that of an oxidized surface from $-130 \mathrm{mV}$ to $245 \mathrm{mV}$ depending on the cleaning treatment used [21]. For an HCL clean, for example, they reported a change in surface contact potential of $-100 \mathrm{mV}$, while for an $\mathrm{H}_{3} \mathrm{PO}_{4}$ clean, the change in surface contact potential was $-130 \mathrm{mV}$ and domain boundaries and threading dislocations had a $\sim 30 \mathrm{mV}$ lower surface contact potential than the surrounding GaN [21].The effect of wet-chemistry surface treatments will be discussed in more detail later.

The effect that device processing methods can have on surface band bending was explored by Cho et al. [22], who measured surface band bending in $n$-type $\mathrm{GaN}$ by KPFM before and after a reactive ion etch (RIE). They observed an increase from $1.0 \mathrm{eV}$ to $1.4 \mathrm{eV}$ of upward band bending following the RIE and suggested that near surface defects are created during the RIE treatment.

EFM and KPFM have been employed for electrical characterization of IIInitrides surfaces, films and heterostructures. Methods have been established for measuring surface potentials and calculating surface state densities, bare surface barrier heights and band bending. Bridger et al. [16] and Koley et al. [24] demonstrated that quantitative information can be obtained by measurements in air, and Hsu et al. [21] demonstrated that these measurements depend strongly on the surface prehistory. Ultimately, for measurements made in air, the surface condition following a treatment might vary with time, and differentiating effects due to band bending and surface dipoles related to screening charge is difficult, suggesting that further advancements can be made by performing measurements in vacuum or a controlled environment.

\section{Investigation of Defects by SPM}

The potential of SPM for the measurement of electrical properties of nanoscale structures such as quantum dots and the variation of electrical properties due to 
differences in thickness or the presence of defects has only recently been tapped. SPM techniques can be used to measure potentials of surfaces and are ideally suited for measuring the local electronic properties near defects and the charge associated with defects. Controlling and eliminating defects in III-nitride materials is a topic of great interest, as defects can degrade device performance or make them inoperable. In this section, the role of SPM in investigating defects in III-nitride materials is explored.

One of the main challenges for $\mathrm{GaN}$-based technologies has been the large number of extended defects such as screw and threading dislocations in the material. Many such defects are attributed to the lattice mismatch between $\mathrm{GaN}$ and conventional substrates [60]. While recently there have been a number of technological advances in developing substrates for epitaxial III-nitride growth (such as bulk [61] and hydride vapor phase epitaxy freestanding $\mathrm{GaN}$ films [62], seeded growth of AIN [63], etc.), the earliest attempts at growth were performed on commonly used substrates such as sapphire, $\mathrm{SiC}$ and $\mathrm{Si}$, which have a significant lattice mismatch with $\mathrm{GaN}$. This lattice mismatch leads to strain (and also to piezoelectrically induced charge), and strain relaxation leads to dislocations. A high density of dislocations is detrimental for device lifetime and performance. Dislocations could induce carrier scattering [64], non-radiative recombination [65], or lead to high reverse-bias leakage $[39,66]$. In addition, some types of defects are charged while others act as leakage paths. The density and type of defect is very dependent on growth conditions. SPM-based techniques provide a unique combination of nanoscale resolution and electrical characterization and are ideally suited to identify defects and to correlate these defects with local variations in electrical properties. Motivated by the fact that understanding defects can help to eliminate them, the ways in which electrical-based SPM techniques, SCM, EFM, KPFM, and C-AFM have been used to identify the type and charge nature of defects in III-nitride materials are now explored.

As mentioned in the introduction, Hansen et al. [12] employed SCM, which utilizes a tip-generated ac field to cause free carriers to be attracted and repelled by the tip for two-dimensional imaging of carrier dynamics in GaN films. Topography and SCM images are shown in Figure 5(a) and 5(b), respectively. Figure 5(c) shows topography with an overlay of the inverted-contrast SCM image. In this manner,
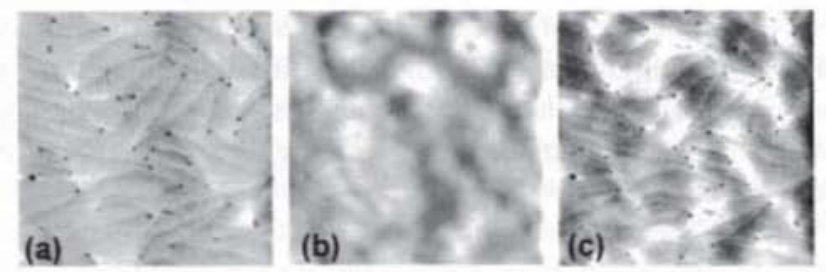

FIGURE 5. Topography (a) and SCM (b) images of the same $2.5 \times 2.5-\mu \mathrm{m}^{2}$ area GaN surface and (c) topography with reverse-contrast SCM image overlaid to highlight the spatial correlation between (a) and (b). Reprinted with permission from [12]. Copyrigb 1998, American Institute of Physics. 


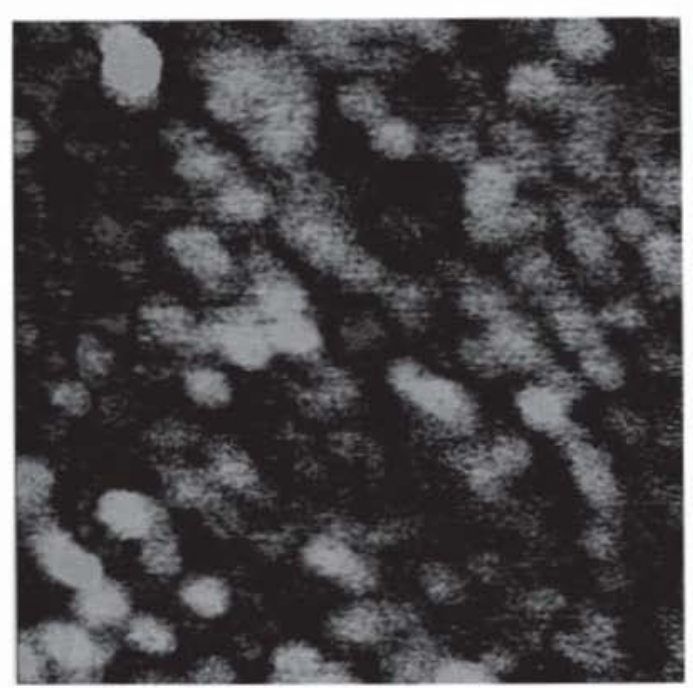

FIGURE 6. SCM image of a $1.0 \times$ $1.0 \mu \mathrm{m}^{2}$ area of an InGaN surface showing indium-rich aggregation domains (bright regions) as small as $50 \mathrm{~nm}$. Image courtesy of Veeco Instruments, Santa Barbara, CA. (See also Plate 9 in the Color Plate Section.)

they demonstrated a correlation between threading dislocations and a reduction in SCM contrast (i.e., a reduction in the change of capacitance with applied voltage (lower $d C / d V)$ ). Furthermore, they observed a shift to positive voltage in local $C-V$ curves measured near the dislocations, suggesting the presence of negative charge. As Hansen et al. [12] point out, however, it is possible that the strain fields surrounding the defects and/or the piezoelectric fields associated with these strain fields could also lead to a voltage shift in the $C-V$ curves.

Zhou et al. [14] employed SCM to characterize InGaN/GaN quantum wells grown by metalorganic chemical vapor deposition. They attributed the variations in carrier concentration observed in the SCM images to monolayer fluctuations in the subsurface InGaN layer. A 2-monolayer variation in InGaN thickness can shift the threshold voltage by $0.6 \mathrm{~V}$. Therefore, the ability of SPM-based techniques to measure variations in electrical properties on the nanoscale can extend to subsurface regions. The aggregation of indium is a common issue for $\mathrm{InGaN}$ growth with high In concentration [67]. Both SCM (Figure 6) and C-AFM [37] have been employed to characterize In-rich regions on the nanoscale. In Figure 6 , local nanometer scale variations in $d C / d V$ are observed, which are attributed to In-rich aggregation domains.

Hsu et al. [15] also employed SCM (C-AFM and KPFM) to investigate the difference in electrical properties of lateral epitaxially (LEO) overgrown GaN, wings (triangular regions formed by intersecting growth planes) and coalescence (where the wings meet) regions in LEO GaN. SCM and C-AFM indicated changes in carrier concentration ( $n_{\text {wing }}>n_{\text {coalescence }}>n_{\text {LEO }}$ ), while KPFM highlighted differences in impurity incorporation and defect structure [15].

Charge rearrangement near defects due to strain relaxation in both Ga- and $\mathrm{N}$-face $\mathrm{GaN}$ were investigated by Bridger et al. [16] using EFM. In N-face GaN, 


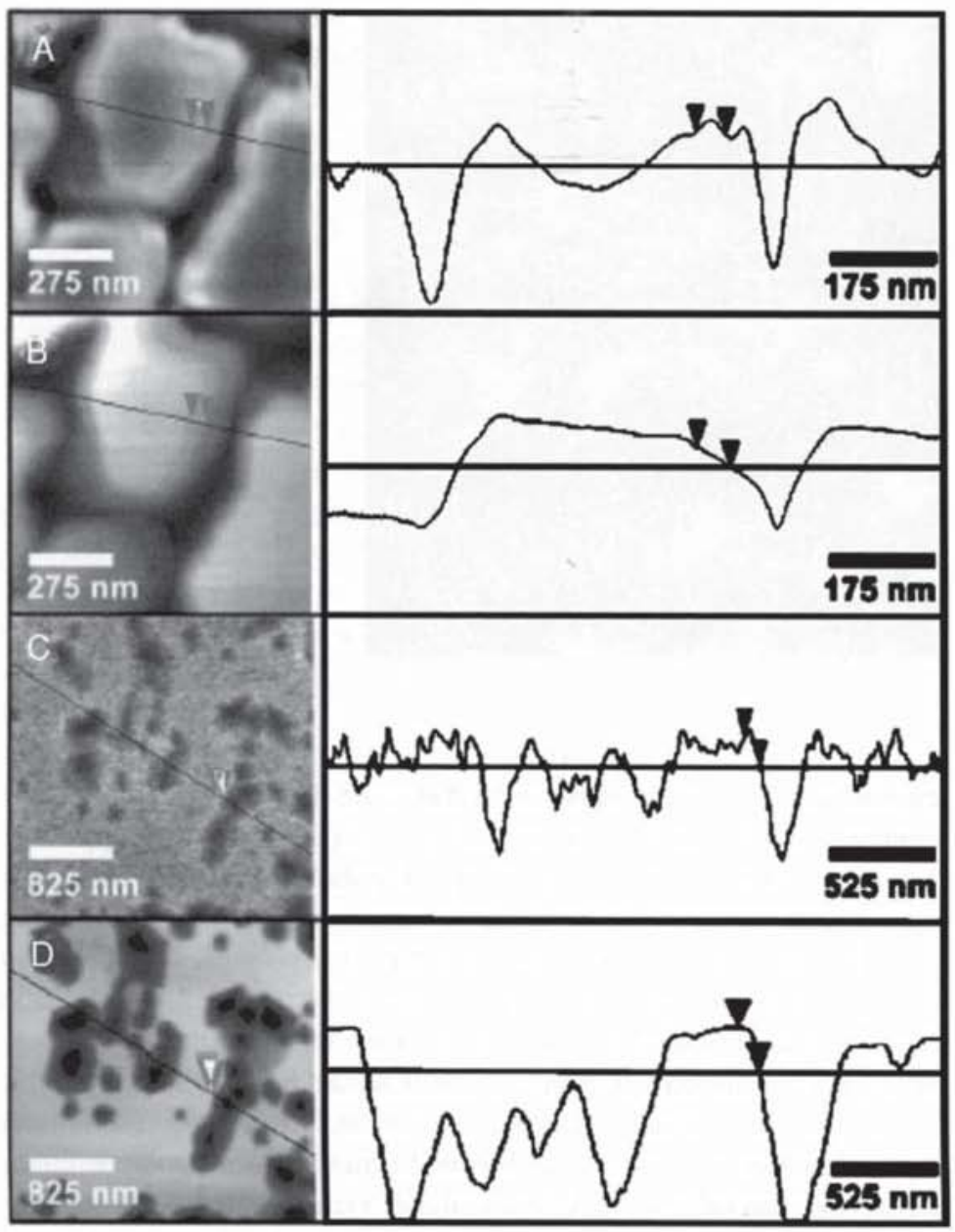

FIGURE 7. (a) EFM and (b) topography images (and associated line profiles) of N-face GaN demonstrating charge accumulation near the edge of steps, and (c) EFM and (d) topography images (and associated line profiles) of $\mathrm{Ga}$-face $\mathrm{GaN}$ showing that charge accumulates near the edge of hexagonal pits. The arrows represent the spatial extent $(60 \mathrm{~nm})$ of the screening charge associated with the defect structure. Reprinted with permission from [16]. Copyright 1999, American Institute of Physics.

charge accumulates at the edge of steps, while in $\mathrm{Ga}$-face $\mathrm{GaN}$, charge accumulates near the edge of hexagonal pits, as shown in Figure 7. In both cases, they found the charge rearrangement to occur over $60 \mathrm{~nm}$, which is approximately equal to the calculated Debye length (electron concentration $\geq 10^{15} \mathrm{~cm}^{-3}$ ), suggesting that the observed charge is truly a screening charge.

KPFM has also been used to determine defect type. Ku et al. [31] measured lower $E_{F}$ by $0.2 \mathrm{eV}$ on $\mathrm{V}$-shaped defects on $\mathrm{AlGaN} / \mathrm{GaN}$ films, reflecting characteristics 

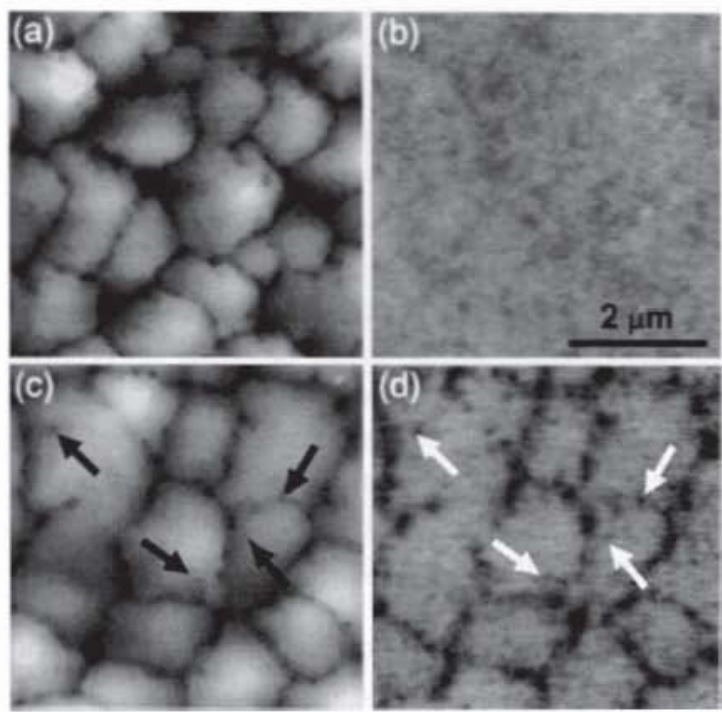

FIGURE 8 . (a) $5 \times 5 \mu^{2}$ topography and (b) KPFM images of an oxidized $\mathrm{GaN}$ surface, and (c) topography and (d) KPFM images of a different area immediately after the sample was cleaned in $160^{\circ} \mathrm{C} \mathrm{H}_{3} \mathrm{PO}_{4}$ for $5 \mathrm{~min}$ utes. Dark regions in (d) indicate the presence of excess negative charge and correspond to domain boundaries or pits in (c) as indicated by arrows. Reprinted with permission from [21]. Copyright 2002, American Institute of Physics.

of acceptor type defects. Koley et al. [23] observed negatively charged dislocations in $n-\mathrm{GaN}$ and $\mathrm{AlGaN}$ surface potential variations of $0.1-0.2 \mathrm{~V}$ and $0.3-0.5 \mathrm{~V}$, respectively. In addition, Choia et al. [32] measured negatively charged extended dislocations with $0.04-0.2 \mathrm{~V}$ higher potential than the surrounding region.

Hsu et al. [21] demonstrated a surface potential dependence on surface treatment, as mentioned previously. Topography and KPFM images of a GaN surface before and after a hot $\mathrm{H}_{3} \mathrm{PO}_{4}$ surface treatment are shown in Figure 8(a,b) and Figure 8(c,d), respectively. Interestingly, they observed a potential variation associated with defects only after cleaning the $\mathrm{GaN}$ surface in hot $\mathrm{H}_{3} \mathrm{PO}_{4}$. Using KPFM, a lower surface potential was detected near dislocations located at domain boundaries, which is consistent with a local excess of fixed negative charge. They did not observe KPFM contrast near screw dislocations, suggesting screw dislocations might have gap states near the conduction band edge.

Not only is KPFM a function of surface treatment, but it also can be modified by ultraviolet (UV) illumination, since photogenerated carries will redistribute to screen electric fields near the surface. This technique is often called surface photovoltage microscopy [65]. Using super-band-gap photons, Bozek et al. [28] observed nano- and microscale variations which they attributed to threading dislocations with a screw component and strain dislocations, respectively. Simpkins et al. [35] correlated C-AFM with KPFM after UV illumination to demonstrate that dislocations exhibit decreased conductivity and larger decreases in KPFM after illumination. They attribute this result to $\mathrm{Mg}$ segregation to dislocation cores. Previously, Bridger et al. [18] also observed a dependence on illumination and further demonstrated that KPFM can be modified by the external application of strain.

The relation between surface potential and dislocations in $\mathrm{GaN}$ films with different doping levels has been explored by Krtschil et al. [29,30]. Using KPFM, 
they determined that the defect is either negatively charged or neutral depending on the type of doping atoms in the layers. They proposed that the dislocations are decorated by an accumulation of charge.

In the final part of this section, we explore how C-AFM can be used to establish a correlation between defect type and leakage current. High reverse bias gate leakage limits device performance, and C-AFM has been employed by several groups to determine if defects contribute to the leakage, and to identify electrically active defects. Hsu et al. [39] used C-AFM to measure reverse bias leakage in GaN films and found that non-zero current was detected primarily on hillocks, suggesting that leakage occurs primarily at dislocations with a screw component. In addition, two samples were examined which had a similar total dislocation density but with screw dislocation densities that differed by an order of magnitude [38]. They found that the sample with a higher density of screw dislocations had a higher density of reverse-bias leakage paths, corroborating that screw dislocations have a greater impact on leakage than do edge or mixed dislocations.

More recently, several other groups have also employed C-AFM for current mapping of GaN films. Dong [44] attributed a reduction in leakage current as measured by C-AFM to the incorporation of hydrogen during growth. Pomarico et al. [45] reported increased conduction on off-axis facet planes. Using KPFM, $\mathrm{Gu}$ et al. [33] also found etched pits and as-grown islands to have lower surface potential, higher workfunction, and increased electrical activity. Spradlin et al. [46] observed a correlation between screw dislocations associated with hillocks and increased leakage current. Furthermore, they investigated both forward and reverse current conduction and measured local $I-V$ curves on these dislocations, revealing a Frenkel-Poole mechanism for forward conduction. On the other hand, Shiojima et al. [43] employed the tip as a probe to measure current-voltage characteristics on submicron Schottky contacts on $n-\mathrm{GaN}$ and found that dislocations did not affect $I-V$ characteristics.

Simpkins et al. [35] have taken electrical characterization of defects by SPM one step further, employing KPFM and C-AFM of the same surface in order to establish a direct correlation between leakage current and the charge state of these leakage paths as shown in Figure 9. The analysis demonstrated that threading dislocations are negatively charged and do not contribute to leakage current, while pure screw type dislocations are not charged and are indeed the source of leakage current. Thus, SPM techniques allow establishment of a direct correlation between charge and defect structure.

SPM-based techniques have even offered scanning modification methods to reduce the effect of leakage current in Schottky diodes. Miller et al. [41] also observed dislocation-related leakage paths in AlGaN/GaN heterostructures. In addition, they demonstrated that the process of scanning with a voltage applied between the tip and sample can lead to the formation of an insulating layer (of gallium oxide) [42] over the dislocation. This layer grows to a thickness of 2-3 $\mathrm{nm}$ and eventually blocks the leakage current. As evidence, they measured $I-V$ characteristics of Schottky diodes fabricated on an AFM-modified area, and found that the surface modification reduced the reverse-bias leakage by a factor of two. 

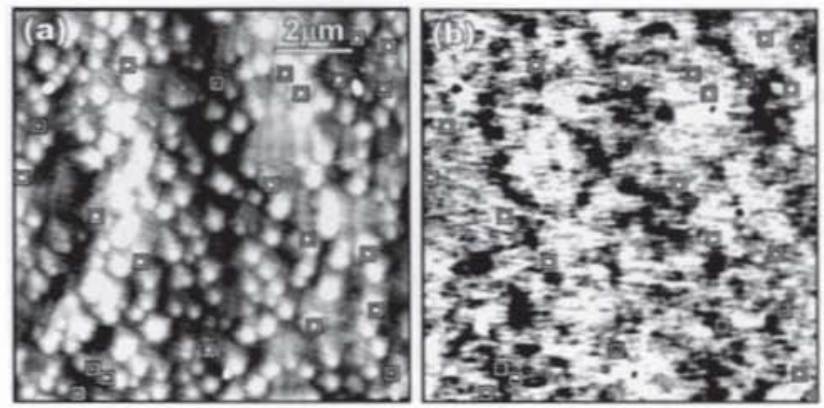

FIGURE 9. (a) Topography and (b) surface potential of a $10 \times 10 \mu \mathrm{m}^{2}$ area of a GaN surface demonstrating the lack of correlation between leakage paths (highlighted by squares) and negatively charged features. Reprinted with permission from [24]. Copyright 2003, American Institute of Physics.

It has been demonstrated that SPM can be used to identify defects, to measure the charge associated with defects, and to modify the surface and block leakage associated with defects. One future challenge would be to use SPM-based techniques to measure the charge distribution and electrical properties near a defect in an operating III-nitride device.

\section{Measurement of Polarity Effects by SPM}

For polarity-based devices, one of the most detrimental defects is an inversion domain-a region within the film that has opposite polarity. Devices based on polarization effects such as HEMTs depend on uniform polarity, and the presence of inversion domains limits the charge density that can be achieved, while the density of inversion domains limits the size of the devices that can be fabricated. SPM techniques, such as PFM and EFM, can be used to locate these inversion domains and to measure the density of defects, thus playing an important role in improving device performance and reliability. As discussed in the introduction, the surface charge and hence the screening charge is intrinsically related to the polarity. Therefore, any discussion of surface charge or polarization screening relates to polarity effects. Thus the distinction between the measurement of electronic properties and polarity effects is somewhat arbitrary. In this section, we distinguish between the two by focusing on the determination of polarity and on polarity screening effects.

The determination of polarity has long been a challenge for the III-nitride community $[9,10]$. While etching can reveal the presence of inversion domains, the method is destructive $[69,70]$. In addition, while the presence of inversion domains can often be deduced from growth conditions and surface topography, these techniques infer polarity rather than measure it directly.

Scanning probe techniques, with their abilities to measure surface charge, surface potential, and piezoelectric response (piezoresponse) to an applied field, can 
FIGURE 10. (a) Topography and (b) EFM of Ga- and N-face regions, half of which are covered by $\mathrm{Pt}$ (right side of dotted line).
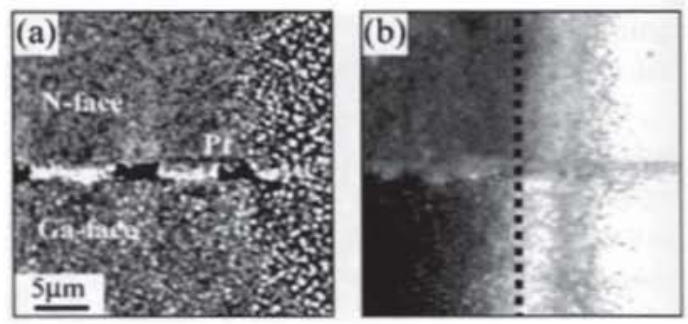

be used to determine polarity. In particular, EFM is a measure of the net surface charge and the polarity can be deduced if the screening charge is known. Figure $10(a, b)$ shows topography and EFM, respectively, of Ga- and $\mathrm{N}$-face regions of a GaN-LPH sample, the right half of which is covered by Pt. Note that while no contrast exists in the EFM image across the inversion domain boundary on the Pt top electrode (suggesting that the metal effectively screens the surface charge), there is polarity-dependent contrast between the two domains in the uncoated region.

Similarly, KPFM can potentially be used to deduce polarity since the contact potential is expected to vary for different polar faces [34]. However, the value obtained for contact potential difference depends strongly on the surface condition $[19,25]$. KPFM and the associated topographic image of a $10 \times 10 \mu \mathrm{m}^{2}$ area of a GaN-LPH surface are shown in Figure 11(a) and 11(b), respectively, demonstrating
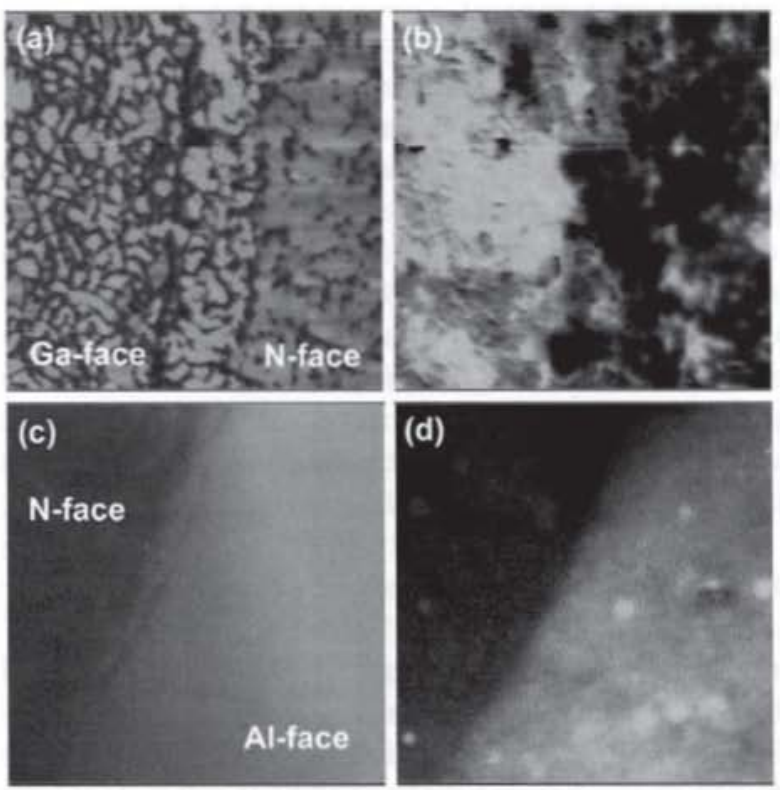

FIGURE 11. (a) Topography and (b) KPFM of a $10 \times 10 \mu \mathrm{m}^{2}$ area of a GaN-LPH surface. (c) Topography and (d) KPFM images $\left(15 \times 15 \mu \mathrm{m}^{2}\right)$ of an AlN bulk crystal. GaN sample courtesy of O. Ambacher and R. Dmitrov. AIN sample courtesy of R. Dalmau and Z. Sitar. 


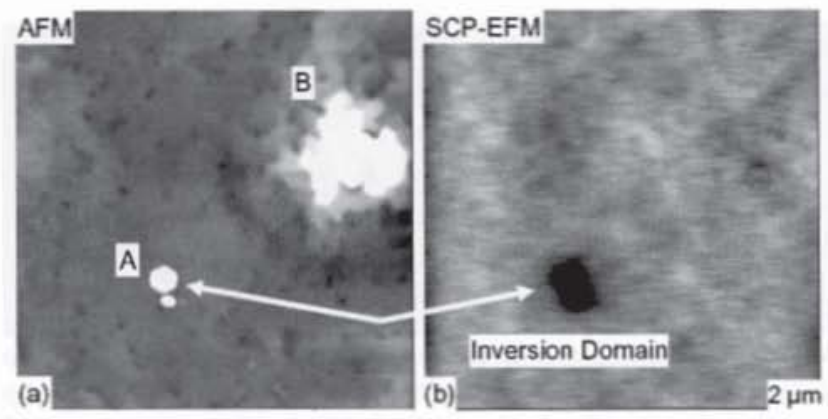

FIGURE 12. (a) Topography and (b) KPFM of a GaN surface showing an inversion domain. Reprinted with permission from [19]. Copyright 2001, American Institute of Physics.

that $\mathrm{Ga}$ - and $\mathrm{N}$-face regions have opposite KPFM contrast related to the polarity. In Figure 11(c) and 11(d), topography and KPFM images of a $15 \times 15 \mu \mathrm{m}^{2}$ area on the surface of an AIN bulk crystal are presented, also demonstrating polaritydependent KPFM contrast. Polarity contrast can also be observed by KPFM on the nanoscale, as shown in Figure 12, which illustrates an inversion domain in a $\mathrm{GaN}$ thin film.

Inverted domains can also be imaged by another SPM technique, PFM, which measures the electromechanical response of the sample when electrostatic forces are minimized (by using a stiff cantilever). In this manner, PFM can be used as a direct measure of polarity [71,72]. In PFM, originally developed for delineation of ferroelectric domains, a periodic electrical bias is applied to a conductive SPM tip, resulting in a periodic displacement of the surface that can be measured with sub-Angstrom precision [73]. The strength and direction of the local electromechanical response reveals the amplitude and phase, respectively, of the subsequent cantilever oscillations. Whereas with EFM the tip deflection is due to the electrostatic interaction between the tip and the net surface charge, with PFM, the tip deflection is due to the mechanical surface displacement and is related to the bulk properties (i.e., sample crystallographic orientation and polarization direction) as illustrated in Figure 13. Furthermore, unlike the piezoresponse of perovskite ferroelectrics (with well-defined crystallographic orientation [74,75]) which can be used to evaluate spontaneous polarization [76], for pyroelectric materials such as the III-nitrides, the magnitude of the spontaneous polarization is a second order correction to the piezoresponse $[77,78]$.

PFM has been employed to determine the polarity in III-nitride thin films and bulk crystals [47-49]. PFM of a GaN-based LPH is shown in Figure 14. The phase contrast is directly related to the polarity of the GaN, while the PFM magnitude is nearly the same for the two opposite domains. Figure 15(a) shows PFM of an inversion domain in a sputtered AIN thin film. From the PFM amplitude and phase images (Figure 15(b,c)), it is evident that the two regions have similar piezoresponse and different phase response, suggesting that the composition is the same, but with different orientation. These images demonstrate the ability of PFM 

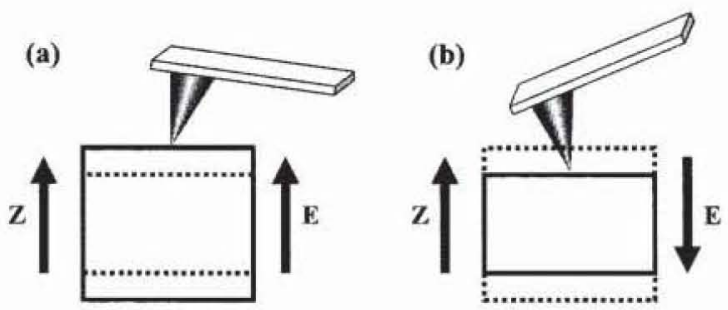

FIGURE 13. A schematic demonstrating the response of a material $\left(d_{33}>0\right)$ to an applied electric field (a) parallel to the Z-axis and (b) antiparallel to the Z-axis. By convention, an electric field applied parallel to the Z-axis will cause a piezoelectric material to expand for $d_{33}>0$. We define in-phase to mean a sample expands when the applied field is generated by a positively biased tip. A sample with $d_{33}>0$ will therefore oscillate out-of-phase with the modulation voltage. The sign of $d_{33}$ is related to the crystallographic orientation of the sample, which also defines the direction of spontaneous polarization in III-nitrides. Therefore, a measurement of the sign of $d_{33}$ (from PFM phase) is also a measurement of crystal polarity and polarization direction. Dotted line represents sample shape before application of electric field.

to determine polarity and identify inversion domains. PFM offers a significant advantage compared to macroscopic techniques in measuring the properties of piezoelectric films since PFM can resolve nanometer variations in the piezoelectric properties of a sample. In addition, PFM directly measures electromechanical response, a material property essential to the employment of piezoelectrics such as III-nitrides in microelectromechanical systems. PFM has also been used to measure the magnitude of the piezoelectric response and thus the effective longitudinal piezoelectric coefficient, $d_{33}$, of III-nitride thin films, and the results are consistent with literature values [49].

For measurements of $d_{33}$ using epitaxial GaN/AIN and AIN layers prepared by organo-metallic vapor phase epitaxy on $\mathrm{SiC}$, a frequency of $1 \mathrm{kHz}$ was used and
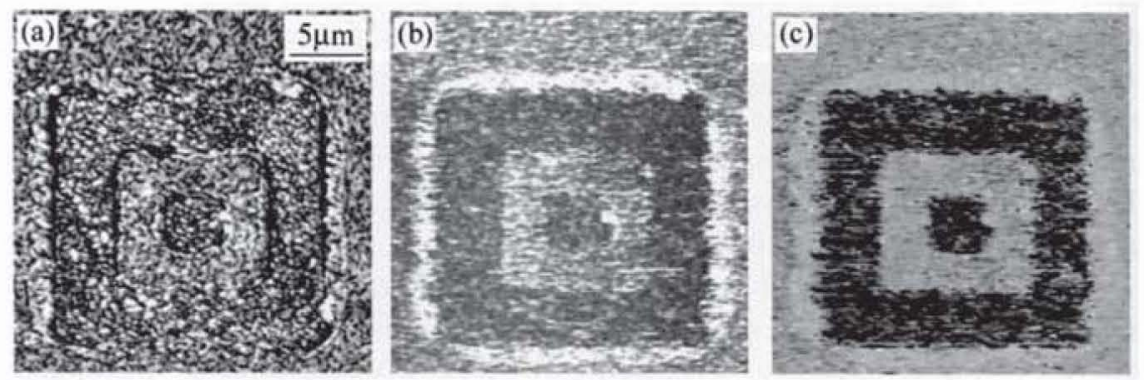

FIGURE 14. Topographic (a), PFM magnitude (b) and PFM phase (c) images of a GaN-based $\mathrm{LPH}$. The innermost $5 \times 5 \mu \mathrm{m}^{2}$ square is a Ga-face region. The $\mathrm{N}$-face region has a higher piezoresponse magnitude as indicated by contrast, and there is a sharp contrast difference in the phase image, demonstrating inversions in film polarity. Reprinted with permission from [47]. Copyright 2002, American Institute of Physics. 

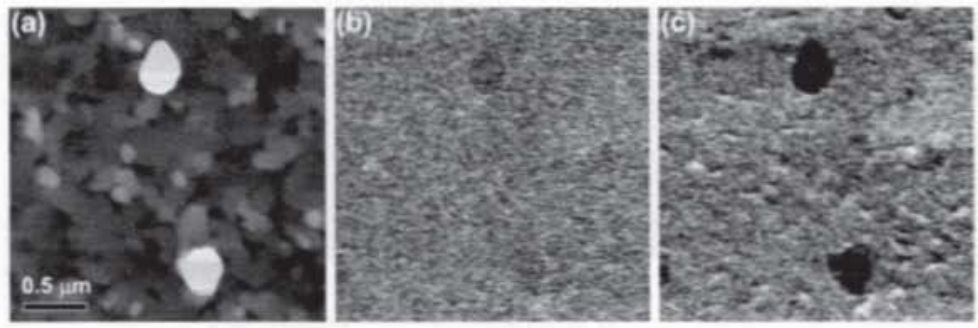

FIGURE 15. (a) Topography, (b) PFM magnitude, and (c) PFM phase images of AlN/Si antiphase domains in a predominantly $\mathrm{N}$-face sample. Reprinted with permission from [48].

a modulation voltage $(0.5-5.0 \mathrm{~V})$ was applied to 80 -nm-thick Pt top electrodes of 100 and $200 \mu \mathrm{m}$ diameters. In this case, the generated field is uniform and the quantitative determination of $d_{33}$ becomes possible [71,75]. The electrodes should be small enough to minimize any wafer-bending that may occur upon application of the electric field [79].

In order to determine the effective piezoelectric coefficient, once a piezoresponse magnitude image was obtained, a histogram was generated, and the peak value determined. For GaN/AIN/SiC films we observe $d_{33}=2 \pm 1 \mathrm{pm} / \mathrm{V}$, and for $\mathrm{AlN} / \mathrm{SiC}$, we observe $3 \pm 1 \mathrm{pm} / \mathrm{V}$. The standard deviation measured from the image histogram indicates the variation in piezoelectric properties across the surface of a film.

The results of these microscopic measurements can be compared to data of macroscopic methods such as interferometric techniques. Using an interferometric method, Lueng et al. [80] reported $d_{33}=3.9 \pm 0.1 \mathrm{pm} / \mathrm{V}$ for $\mathrm{AlN} / \mathrm{Si}(111)$ and $2.7 \pm 0.1 \mathrm{pm} / \mathrm{V}$ for both $\mathrm{GaN} / \mathrm{AlN} / \mathrm{Si}(100)$ and $\mathrm{GaN} / \mathrm{AlN} / \mathrm{Si}(111)$ heterostructures (all films were prepared by MBE). Guy et al. [81] reported $2.0 \pm 0.1 \mathrm{pm} / \mathrm{V}$ for polycrystalline $\mathrm{GaN} / \mathrm{Si}(100)$ grown by laser assisted chemical vapor deposition (CVD); $2.8 \pm 0.1 \mathrm{pm} / \mathrm{V}$ for single crystal GaN/SiC grown by hydride vapor phase epitaxy; and $3.2 \pm 0.3 \mathrm{pm} / \mathrm{V}$ and $4.0 \pm 0.1 \mathrm{pm} / \mathrm{V}$ for polycrystalline $\mathrm{AlN} / \mathrm{Si}(100)$ heterostructures grown by plasma-assisted and laser-assisted CVD, respectively.

In order to understand the screening mechanism in III-nitrides and the role screening plays on band bending and surface potential, EFM and KPFM have been employed by Rodriguez et al. [20] on the same surface before and after an HCL surface treatment which is known to remove oxide. In measurements of the as-received sample, the KPFM revealed a surface potential (relative to Pt) of $0.3 \mathrm{~V}$ for the $\mathrm{Ga}$ face and $0.9 \mathrm{~V}$ for the $\mathrm{N}$-face for a potential difference of $0.6 \mathrm{~V}$ as shown in Figure 16. Following an $\mathrm{HCl}$ treatment, the surface potential did not change for the $\mathrm{Ga}$-face and decreased to $0.6 \mathrm{~V}$ for the $\mathrm{N}$-face (Figure 16(c)).

Under the assumptions that (1) there is a dc bias that equalizes the force on the tip, and (2) that $\partial C_{t} / \partial z$ has the same magnitude but opposite sign for the polar faces, the difference in surface charge density between the polar faces can 


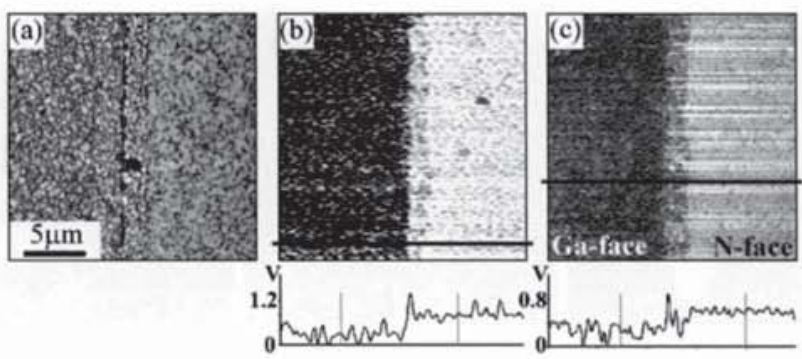

FIGURE 16. (a) Topography of a $20 \times 20-\mu \mathrm{m}^{2}$ area, (b) KPFM (with line profile) of a GaNLPH prior to surface treatment, and (c) KPFM of the same area after surface treatment. Reprinted with permission from [20]. Copyright 2005, American Institute of Physics.

be obtained. From Eq. (1), it can be shown that:

$$
\left|\sigma^{N}\right|-\left|\sigma^{G a}\right|=\frac{\varepsilon_{0}(1+\kappa)}{C_{t}}\left(\frac{\partial C_{t}}{\partial z}\right)\left(2 V_{d c}^{\prime}-V_{s}^{N}-V_{s}^{G a}\right),
$$

where $V_{d c}^{\prime}$ is the value of dc bias that equalizes the forces.

The EFM $\left(V_{d c}=0\right)$ of the same area before the surface treatment revealed that the electrostatic force on the tip is larger on the $\mathrm{N}$-face than the Ga-face GaN. The EFM phase measurements indicated that the net surface charge (superposition of polarization and screening charge) is positive for the $\mathrm{N}$-face surface and negative for the $\mathrm{Ga}$-face surface. Following the surface treatment, the electrostatic force for the $\mathrm{N}$-face further increased, while the EFM phase measurements revealed that the net surface charge remained positive for the $\mathrm{N}$-face surface and negative for the Ga-face surface.

Figure 17(a-c) shows EFM phase images and Figure 17(d-f) shows EFM magnitude images for tip biases of $0 \mathrm{~V}, 1 \mathrm{~V}$, and $2 \mathrm{~V}$, respectively, of the as-received
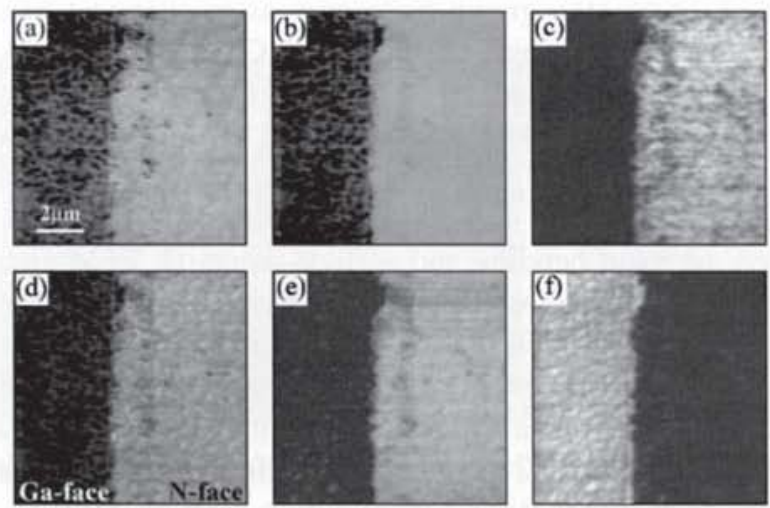

FIGURE 17. (a-c) EFM phase and (d-f) EFM magnitude images of a $10 \times 10-\mu \mathrm{m}^{2}$ region on the GaN-LPH sample with a dc bias of $0 \mathrm{~V}, 1 \mathrm{~V}$ and $2 \mathrm{~V}$, respectively. Reprinted with permission from [20]. Copyright 2005, American Institute of Physics. 


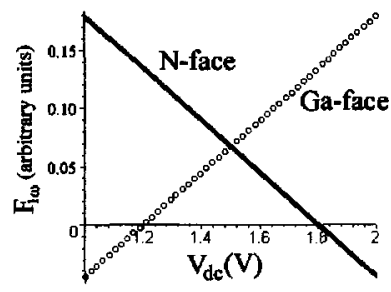

FIGURE 18. Plot of electrostatic force as a function of applied dc bias demonstrating that the electrostatic force on the tip is greater for the $\mathrm{N}$-face $\mathrm{GaN}$ when no bias is applied and greater for the Ga-face $\mathrm{GaN}$ when the bias is greater than 1.5 V. Reprinted with permission from [20]. Copyright 2005, American Institute of Physics.

surface. The results indicate that a tip bias of $1.5 \mathrm{~V}$ equalizes the electrostatic force on the tip from the $\mathrm{Ga}$ - and $\mathrm{N}$-face regions, and the contrast reverses for a tip bias above $1.5 \mathrm{~V}$.

At $0 \mathrm{~V}$ bias, the tip responds to a net negative charge on the Ga-face $\mathrm{GaN}$ and a net positive charge on the $\mathrm{N}$-face $\mathrm{GaN}$. At this bias, the magnitude of the EFM signal indicates that the net surface charge on the $\mathrm{N}$-face is greater, suggesting that the screening charge (both external and internal) is greater on the Ga face. If both faces are assumed to have roughly the same degree of internal screening, the results suggest the Ga-face surface has more adsorbed charge. As the bias $\left(V_{d c}\right)$ is increased, the second term in Eq. (1) is reduced for the $\mathrm{N}$-face but increased for the Ga-face, which explains the change in magnitude contrast. This is demonstrated graphically in Figure 18.

Using the method of image charge approach and assuming the manufacturer specified tip radius $R=50 \mathrm{~nm}$ and the chosen operating tip-sample distance $z=70 \mathrm{~nm}, 7 \times 10^{-18} \mathrm{~F}$ and $-1.6 \times 10^{-11} \mathrm{~F} / \mathrm{m}$ are obtained for $C_{t}$ and $\partial C_{t} / \partial z$, respectively $[53,82]$. Using Eq. (3), the net surface charge density difference can be determined to be $\left|\sigma^{N}\right|-\left|\sigma^{G a}\right|=3.6 \pm 0.4 \times 10^{-5} \mathrm{C} / \mathrm{m}^{2}$ prior to the surface treatment and $\left|\sigma^{N}\right|-\left|\sigma^{G a}\right|=6.2 \pm 0.8 \times 10^{-5} \mathrm{C} / \mathrm{m}^{2}$ after the surface treatment, indicating a net increase in the surface charge density difference between faces. Since the surface potential for the Ga-face remained the same, the change in the difference in surface charge density is attributed to the N-face only. The corresponding increase in surface charge density for the $\mathrm{N}$-face is roughly $(1.6 \pm 0.5)$ $\times 10^{10}$ electrons $/ \mathrm{cm}^{2}$, corresponding to a small fraction of the bound polarization charge $\left(\sigma_{P}=2.1 \times 10^{13} \mathrm{~cm}^{-2}\right)$. This slight increase of the surface charge has a negligible effect on the percentage of screening, which is close to $99.9 \%$ in both cases. Since a reduction in net (positive) surface charge is observed and the electrostatic force on the tip changes only for the $\mathrm{N}$-face $\mathrm{GaN}$, it is reasonable to conclude that the surface treatment added adsorbed charge to the $\mathrm{N}$-face regions.

The band bending at the as-received $\mathrm{N}$-face surface is initially flat or bent slightly upward and increases as a result of the $\mathrm{HCl}$ treatment. Because the deduced net charge is not large enough to account for the observed change in surface potential, surface states or defects must be present near the surface to receive the excess negative charge to allow the upward band bending. Since these measurements were performed in air as opposed to a vacuum environment, it is impossible to establish the relative contribution from the band bending and surface dipole.

EFM and KPFM techniques were used to evaluate the surface charge density of a GaN-LPH by determining the potential difference and the tip bias that 
equated the electrostatic force due to both polar faces. Unlike most ferroelectric oxide surfaces, for which internal screening is secondary to screening by adsorbed species [83], GaN is primarily screened by internal charge $\left(N_{d}=4.1 \times 10^{17} \mathrm{~cm}^{-3}\right)$ [84]. It has been found that the Ga-face surface was unaffected by the $\mathrm{HCl}$ surface treatment, while the surface potential of the $\mathrm{N}$-face $\mathrm{GaN}$ was reduced in the process.

\section{Summary and Outlook}

In this review, the impact of electrical characterization by SPM on the III-nitride material system has been presented. From the determination of polarity in films and bulk crystals alike, to the measurement of $d_{33}$, and to the elucidation of the screening mechanism in Ga- and N-face GaN, it has been established that SPMbased electrical characterization methods can be used to investigate polarity effects in III-nitrides and nitride-based devices and address critical issues such as band bending, barrier heights, and defect-related degradation effects. SPM techniques have played an important role in the development and advancement of III-nitrides and are certain to continue to have an impact. Information gained by SPM opens the door to the development of novel devices which make use of the polarization in IIInitrides, including HEMTs, capable of employing either 2DEGs or 2 dimensional hole gases, and detection sensors based on changes in measured 2DEG density resulting from changes in adsorbed charge. There are several clear avenues for continued impact. First, vacuum or controlled ambient measurements are needed. Only then can the surface be modified in a controlled way. Second, measurements should be performed on active devices. In this manner, device performance and defect type can be related in an operating device, a possibility first demonstrated by $\mathrm{Hsu}$ et al. [51] using scanning gate microscopy on $\mathrm{AlGaN} / \mathrm{GaN}$ transistors to correlate threading dislocations with lower 2DEG density. Third, SPM is ideally suited to address issues related to III-nitride/ferroelectric heterostructure fabrication and device design, which has recently attracted interest [85-90]. Lastly, while some devices have been developed that exploit the polarization in these materials, the potential has yet to be fully realized. It is intriguing to suggest that the large surface-bound polarization charge in the III-nitrides can be used to drive self-assembly of charged molecules.

Acknowledgments. The authors would like to thank O. Ambacher and R. Dmitrov for the LPH-GaN sample, R. Dalmau and Z. Sitar for the AlN bulk crystal, and V. Lebedev for the AIN on Si thin film. We would also like to acknowledge S. V. Kalinin for providing instrument time to obtain the images in Figure 11 and T. C. Blair for useful discussions. We extend our gratitude to B. S. Simpkins for useful comments related to this chapter and also to J. W. P. Hsu, H. Morkoç, E. T. Yu, and M. G. Spencer for kindly discussing their results. We gratefully acknowledge the support of the Office of Naval Research MURI on Polarization Electronics Contract 
No. N00014-99-1-0729 and the National Science Foundation (Grant No. 0403871, NIRT on Nanopatterned Polar Surfaces and Grant No. DMR02-35632).

\section{References}

1. H. Morkoc, R. Cingolani, B. Gil, Mat. Res. Innovat. 3, 97 (1999).

2. L. F. Eastman, U. K. Mishra, IEEE Spectrum May 2002, pp. 28-33.

3. A. Zoroddu, F. Bernardini, P. Ruggerone, V. Fiorentini, Phys. Rev. B 64, 045208 (2001).

4. S. Strite and H. Morkoc, J. Vac. Sci. Tech. B 10, 1237 (1992).

5. K. M. Yu, Z. Liliental-Weber, W. Walukiewicz, W. Shan, J. W. Ager, III, S. X. Li, R. E. Jones, E. E. Haller, Hai Lu, and William J. Schaff, Appl. Phys. Lett. 86, 071910 (2005).

6. F. Bernardini and V. Fiorentini, Appl. Phys. Lett. 80, 4145 (2002).

7. O. Ambacher, J. Smart, J. R. Shealy, N. G. Weimann, K. Chu, M. Murphy, R. Dimitrov, L. Wittmer, M. Stutzmann, W. Rieger and J. Hilsenbeck, J. Appl. Phys. 85, 3222 (1999).

8. F. Bernardini, V. Fiorentini, D. Vanderbilt, Phys. Rev. B 56, R10024 (1997).

9. E. S. Hellman, MRS Internet J. Nitride Semicond. Res. 3, 11 (1998).

10. M. Sumiya and S. Fuke. MRS Internet J. Nitride Semicond. Res. 9, 1 (2004).

11. U. Karrer, O. Ambacher, M. Stutzmann, Appl. Phys. Lett. 77, 2012 (2000).

12. P. J. Hansen, Y. E. Strausser, A. N. Erickson, E. J. Tarsa, P. Kozodoy, E. G. Brazel, J. P. Ibbetson, U. Mishra, V. Narayanamurti, S. P. DenBaars, and J. S. Speck, Appl. Phys. Lett. 72, 2247 (1998).

13. D. M. Schaadt, E. J. Miller, E. T. Yu, and J. M. Redwing, Appl. Phys. Lett. 78, 88 (2001).

14. X. Zhou, E.T. Yu, D. Florescu, J.C. Ramer, D.S. Lee, E.A. Armour, Appl. Phys. Lett. 85, 407 (2004).

15. J. W. P. Hsu, M. J. Matthews, D. Abusch-Magder, R. N. Kleiman, D. V. Lang and S. Richter, S. L. Gu and T. F. Kuech, Appl. Phys. Lett. 79, 761 (2001).

16. P. M. Bridger, Z. Z. Bandić, E. C. Piquette, and T. C. McGill, Appl. Phys. Lett. 74, 3522 (1999).

17. N. M. Shmidt, V. V. Emtsev, A. S. Kryzhanovsky, R. N. Kyutt, W. V. Lundin, D. S. Poloskin, V. V. Ratnikov, A. V. Sakharov, A. N. Titkov, A. S. Usikov, and P. Girard, Phys. Stat. Sol. (b) 216, 581 (1999).

18. P. M. Bridger, Z. Z. Bandić, E. C. Piquette, and T. C. McGill, J. Vac. Sci. Technol, B 17, 1750 (1999).

19. K. M. Jones, P. Visconti, F. Yun, A. A. Baski, and H. Morko 5 , Appl. Phys. Lett. 78, 2497 (2001).

20. B. J. Rodriguez, W.-C. Yang, R. J. Nemanich, and A. Gruverman, Appl. Phys. Lett. 86, 112115 (2005).

21. J. W. P. Hsu, H. M. Ng, A. M. Sergent, and S. N. G. Chu, Appl. Phys. Lett. 81, 3579 (2002).

22. S.-J. Cho, S. Doan, S. Sabuktagin, M. A. Reshchikov, D. K. Johnstone, and H. Morko $\varsigma$, Appl. Phys. Lett. 84, 3070 (2004).

23. G. Koley and M. G. Spencer, Appl. Phys. Lett. 78, 2873 (2001).

24. G. Koley and M. G. Spencer J. Appl. Phys. 90, 337 (2001). 
25. V. Cimalla and O. Ambacher, M. Eickhoff, C. Miskys, M. Stutzmann, B. J. Rodriguez, R. Nemanich, M. Drakopoulos, and J. Zegenhagen, presented at the International Workshop on Nitride Semiconductors 2002, Aachen, Germany (unpublished).

26. M. I. N. da Silva, J. C. Gonzakesm, and P. E. Russell, Microelec. J. 34, 571 (2003).

27. B. S. Simpkins, D. M. Schaadt, E. T. Yu, and R. J. Molnar, J. Appl. Phys, 91, 9924 (2002).

28. R. Bozek, K. Pakula, and J. Baranowski, Eur. Phys. J. Appl. Phys. 27, 97 (2004).

29. A. Krtschil, A. Dadgar, and A. Krost, J. Cryst. Growth 248, 542 (2003).

30. A. Krtschil, A. Dadgar, and A. Krost, Appl. Phys. Lett. 82, 2263 (2003).

31. C. S. Ku, J. M. Peng, W. C. Ke, H. Y. Huang, N. E. Tang, W. K. Chen, W. H. Chen, and M. C. Lee, Appl. Phys. Lett. 85, 2818 (2004).

32. H. Choia, E. K. Koha, Y. M. Chob, J. Jinc, D. Byunc, and M. Yoona, Microelec. J. 36, 25 (2005).

33. X.-X. Gu, D.-M. Huang, and H. Morkoç, Chin. Phys. Lett. 20, 1822 (2003).

34. M. Losurdo, M. M. Giangregorio, P. Capezzuto, G. Bruno, G. Namkoong, W. A. Doolittle, and A. S. Brown, J. Appl. Phys. 95, 8408 (2004).

35. B. S. Simpkins, E. T. Yu, P. Waltereit, and J. S. Speck, J. Appl. Phys. 941448 (2003).

36. B. S. Simpkins, E. T. Yu, U. Chowdhury, M.M. Wong, T.G. Zhu, D.W. Yoo, and R.D. Dupuis, J. Appl. Phys. 95, 6225 (2004).

37. F. Iwata, S. Chu, A. Sasaki, K. Ishino, A. Ishida, and H. Fujiyasu, J. Appl. Phys. 88, $1670(2000)$.

38. J. W. P. Hsu, M. J. Manfra, R. J. Molnar, B. Heying, and J. S. Speck, Appl. Phys. Lett. 81, 79 (2002).

39. J. W. P. Hsu, M. J. Manfra, D. V. Lang, S. Richter, S. N. G. Chu, A. M. Sergent, R. N. Kleiman, L. N. Pfeiffer, and R. J. Molnar, Appl. Phys. Lett. 78, 1685 (2001).

40. J. W. P. Hsu, M. J. Manfra, S. N. G. Chu, C. H. Chen, L. N. Pfeiffer and R. J. Molnar, Appl. Phys. Lett. 78, 3980 (2001).

41. E. J. Miller, D. M. Schaadt, E. T. Yu, C. Poblenz, C. Elsass, and J. S. Speck, J. Appl. Phys. 91, 9821 (2002).

42. E. J. Miller, D. M. Schaadt, E. T. Yu, X. L. Sun, L. J. Brillson, P. Waltereit, and J. S. Speck, J. Appl. Phys. 94, 7611 (2003).

43. K. Shiojima, T. Suemitsu, and M. Ogura, Appl. Phys. Lett. 78, 3636 (2001).

44. Y. Dong, Appl. Phys. Lett. 86, 1221917 (2005).

45. A. A. Pomarico, D. Huang, J. Dickinson, A. A. Baski, R. Cingolani, H. Morkoc and R. Molnar, Appl. Phys. Lett. 82, 1890 (2003).

46. J. Spradlin, S. Dogan, J. Xie, R. Molnar, A. A. Baski, and H. Morkoc, Appl. Phys. Lett. 84, 4150 (2004).

47. B. J. Rodriguez, A. Gruverman, A. I. Kingon, and R. J. Nemanich, and O. Ambacher, Appl. Phys. Lett. 80, 4166 (2002).

48. B. J. Rodriguez, A. Gruverman, A. I. Kingon, and R. J. Nemanich, J. Cryst. Growth 246, 252 (2002).

49. B. J. Rodriguez, D.-J. Kim, A. I. Kingon, and R. J. Nemanich, Mat. Sci. Soc. Symp. 693, I9.9 (2002).

50. R. Dalmau, R. Schlesser, B. J. Rodriguez, R. J. Nemanich, and Z. Sitar, J. Cryst. Growth 281, 68-74 (2005).

51. J. W. P. Hsu, N. G. Weimann, M. J. Manfra, K. W. West, D. V. Lang, F. F. Schrey, O. Mitrofanov, and R. J. Molnar, Appl. Phys. Lett. 83, 4559 (2003).

52. B. D. Terris, J. E. Stern, D. Rugar, and H. J. Mamin, Phys. Rev. Lett. 63, 2669 (1989).

53. F. Saurenbach and B. D. Terris, Appl. Phys. Lett. 56, 1703 (1990). 
54. M. Nonnenmacher, M. P. O'Boyle, and H. K. Wickramasinghe, Appl. Phys. Lett. 58, 2921 (1991).

55. D. Sarid, Scanning Force Microscopy (Oxford University Press, New York, 1991).

56. A. M. Cowley and S. M. Sze, J. Appl. Phys. 36, 3212 (1965).

57. S. V. Kalinin and D. A. Bonnell, Phys. Rev. B 63, 125411 (2001).

58. U. Karrer, O. Ambacher, and M. Stutzmann, Appl. Phys. Lett. 77, 2012 (2000).

59. T. E. Cook, C. C. Fulton, W. J. Mecouch, K. M. Tracy, R. F. Davis, E. H. Hurt, G. Lucovsky, and R. J. Nemanich, J. Appl. Phys. 93, 3995 (2003).

60. L. Liu and J.H. Edgar, Mat. Sci. Eng. R 37, 61 (2002)].

61. M. Boćkowski, I. Gregory, S. Krukowski, B. Lucznik, M. Wróblewski, G. Kamler, J. Borysiuk, P. Kwiatkowsko, K. Jasik, and S. Porowski, J. Cryst. Growth 274, 55 (2005).

62. C. R. Miskys, M. K. Kelly, O. Ambacher, and M. Stutzmann, Phys. Stat. Sol. C 0, 1627 (2003).

63. R. Schlesser, R. Dalmau, and Z. Sitar, J. Cryst. Growth 241, 416 (2002).

64. D. Jena, A. C. Gossard, and U. K. Mishra, Appl. Phys. Lett. 76, 1707 (2000).

65. S. J. Rosner, E. C. Carr, M. J. Ludowise, G. Girolami, and H. I. Erikson, Appl. Phys. Lett. 70, 420 (1997).

66. E. J. Miller, E. T. Yu, P. Waltereit, and J. S. Speck, Appl. Phys. Lett. 84, 535 (2004).

67. T. Ive, O. Brandt, M. Ramsteiner, M. Giehler, H. Kostial, and K. H. Ploog, Appl. Phys. Lett. 84, 1671 (2004).

68. L. Kronik and Y. Shapira, Surf. Sci. Rep. 37, 1 (1999).

69. J. L. Weyher, S. Müller, I. Grzegory, and S. Porowski, J. Cryst. Growth 182, 17 (1997).

70. D. Li, M. Sumiya, S. Fluke, D. Yang, D. Que, Y. Suzuki, and Y. Fukada, J. Appl. Phys. 90, 4219 (2001).

71. J. A. Christman, R. Woolcott, Jr., A. Kingon, R. J. Nemanich, Appl. Phys. Lett. 73, 3851 (1998).

72. S. V. Kalinin and D. A. Bonnell, Phys. Rev. B 65, 125408 (2002).

73. A. Gruverman, O. Auciello, H. Tokumoto, Ann. Rev. Mat. Sci. 28, 101 (1998).

74. S.V. Kalinin, E. Karapetian, and M. Kachanov, Phys. Rev. B 70, 184101 (2004).

75. M. Alexe and A. Gruverman, Nanoscale Characterization of Ferroelectric Materials (Springer-Verlag, New York, 2004).

76. C. Harnagea, A. Pignolet, M. Alexe, D. Hesse, Integrated Ferroelectrics 44, 113 (2002).

77. J. F. Nye, Physical Properties of Crystals (Oxford Science Publications, 1985).

78. B. J. Rodriguez, 2003. Nanoscale Investigation of The Piezoelectric Properties of Perovskite Ferroelectrics and III-Nitrides. PhD thesis, North Carolina State University, Raleigh, NC.

79. A. L. Kholkin, C. Wuetchrich, D. V. Taylor, and N. Setter, Rev. Sci. Instrum. 67, 1935 (1996).

80. C. M. Lueng, H. L. W. Chan, C. Surya, and C. L. Choy, J. Appl. Phys. 88, 5360 (2000).

81. I. L. Guy, S. Muensit, and E. M. Goldys, Appl. Phys. Lett. 75, 4133 (1999).

82. E.J. Mele, Am. J. Phys. 69, 557 (2001).

83. S. V. Kalinin and D. A. Bonnell, Nano Letters, 4555 (2004).

84. M. Park, J. J. Cuomo, B. J. Rodriguez, W.-C. Yang, R. J. Nemanich, and O. Ambacher, J. Appl. Phys. 93, 9542 (2003).

85. A. Gruverman, W. Cao, S. Bhaskar, S. K. Dey, Appl. Phys. Lett. 84, 5153 (2004).

86. A. Doolittle, G. Namkoong, A. Carver, W. Henderson, D. Jundt, A. Brown, Mat. Res. Soc. 743, 9 (2002).

87. G. Namkoong, K.-K. Lee, S. M. Madison, W. Henderson, W. A. Doolittle, and S. E. Ralph, Appl. Phys. Lett. 87, 171107 (2005). 
88. W. P. Li, R. Zhang, J. Yin, X. H. Liu, Y. G. Zhou, B. Shen, P. Chen, Z. Z. Chen, Y. Shi, R. L. Jiang, Z. G. Liu, Y. D. Zheng, Z. C. Huang, MRS Internet J. Nitride Semicond. Res. 5S1, W11.5 (2000).

89. M. Singh, Y.-R. Wu, J. Singh, Solid St. Elec. 47, 2155 (2003).

90. I. Stolichnov, L. Malin, P. Muralt, and N. Setter, Appl. Phys. Lett. 88, 043512 (2006). 